\title{
Téoros
}

Revue de recherche en tourisme

\section{L'intervention étatique et le tourisme : la perspective fédérale}

\section{Guy Bédard}

Volume 9, numéro 1, mars 1990

Plans et politiques touristiques

URI : https://id.erudit.org/iderudit/1080286ar

DOI : https://doi.org/10.7202/1080286ar

Aller au sommaire du numéro

Éditeur(s)

Université du Québec à Montréal

ISSN

0712-8657 (imprimé)

1923-2705 (numérique)

Découvrir la revue

Citer cet article

Bédard, G. (1990). L'intervention étatique et le tourisme : la perspective fédérale. Téoros, 9(1), 11-14. https://doi.org/10.7202/1080286ar d'utilisation que vous pouvez consulter en ligne.

https://apropos.erudit.org/fr/usagers/politique-dutilisation/ 


\section{L'intervention étatique et le tourisme: la perspective fédérale}

Concevoir, développer, mettre en marché et vendre des rêves et des expériences, voilà l'essentiel du tourisme. Au-delà des rềves, il y a des réalités économiques, sociales et commerciales qui amènent les gouvernements de tous les niveaux à se pencher sur les politiques, programmes ou mesures les plus susceptibles d'appuyer le développement de 1 'industrie touristique.

Le début d'une décennie est un temps propice pour faire le point. C'est dans ce contexte que $\mathrm{s}^{+}$inscrit cet article, qui se veut un rappel des principales politiques et des programmes du gouvernement fédéral qui ont marqué les années ' 80 , que ce soit au plan de la recherche, du marketing ou du développement du produit. Retracer l'évolution est une démarche relativement facile; mais il est plus ardu de cerner les principaux éléments de contexte qui conditionneront l'intervention gouvernementale au cours des années '90 et d'esquisser ce qui pourrait être le rôle de l'État au plan fédéral. Ce cheminement est cependantessentiel dans un contexte économique et fiscal où le rôle du gouvernement est constamment mis au defi.

\section{Les structures continuent de changer}

La première moitié des années ' 80 a été marquée par des changements significatifs au plan des structures et des politiques fédérales touchant le tourisme.

Nous nous rappellerons qu'en 1982 , le gouvernement annonçait la fusion des ministères de l'Industrie et du Commerce (MIC) et de l'Expansion Économique Régionale (MEER). Ceci aeu pour effet de regrouper l'Office canadien du tourisme (OCT) - jusque-lă largement axé sur le marketing du tourisme - et les directions $\mathrm{du}$ MEER, responsables de la gestion des ententes fédérales-provinciales, lesquelles visaient principalement le développement du produit. Donc, un premier grand rapprochement marché-produits'opéra. Du même coup, cette réorganisation transférait au ministère des Affaires extérieures (MAE) les agents du tourisme à l'étranger, responsables de la mise en oeuvre des programmes de marketing de l'OCT. Cette façon de faire $s$ 'inspirait d'une nouvelle politique gouvernementale: le chef d'un bureau canadien à l'étranger était responsable de l'ensemble des activités du Canada dans un pays donné, que ce soit l'immigration, l'investissement, le tourisme, etc.

Cette structure a été modifiée à nouveau en 1988, lorsque le gouvernement mit de l'avant sa politique révisée de développement régional, qui entraînait, entre autres, la création de deux agences, l'une pour la région del'Atlantique et l'autre pour celle de l'Ouest. Les questions de développement régional pour le Québec et l'Ontariodevenaient la responsabilité d'une direction générale du nouveau ministère, Industrie, Sciences et Technologie Canada (ISTC).

C'est ce dernier-né qui est aujourd'hui le point de convergence de la gestion du tourisme au sein du gouvernement fédéral (pour simplifier, nous référerons à Tourisme Canada pour désigner la direction générale du tourisme d'ISTC). Ainsi, Tourisme Canada doit assurer le leadership auprès des autres ministères et agences du fédéral, agir en partnership avec les provinces et l'industrie et appuyer les efforts de ces dernières. La création des agences a amené un nouveau joueur sur l'échiquier touristique fédéral, puisqu'elles ont, entre autres, la responsabilité de mettre de l'avant des mesures aux programmes qui sont susceptibles d'accroître le développement économique des régions. Le tourisme constitue, dans plusieurs régions, un outil de développement de première importance et est par conséquent courtisé par les agences: la mise sur pied, en 1989, du programme de développement des entreprises touristiques (PDET) dans les régions ressources du Québec en est un exemple. 


\section{Le tourisme de demain}

En 1985, dans la foulée des consultations auprès de l'ensemble des intervenants du secteur, sur la base du document Le tourisme de demain: pour une stratégie canadienne du tourisme, une série de mesures/ décisions ont été prises. Elles ont influencé la nature et l'étendue de l'intervention fédérale; mentionnons:

- l'accordde Vancouver sur les principes directeurs;

- le groupe de travail sur les données touristiques qui a débouché sur la création d'un compte satellite pour le tourisme;

- la création de l'Institut canadien de recherche en tourisme (ICRT) affilié au Conference Board du Canada;

- la réalisation d'études majeures sur le marché américain;

- une nouvelle stratégie de marketing sur le marché américain;

- la conclusion d'une nouvelle genération d'ententes fédérales-provinciales avec chacune des provinces.

\section{La recherche}

Confiné exclusivement, au début, à un rôle de soutien aux programmes opérationnels de Tourisme Canada, le programme de recherche a évolué vers une tangente caractérisée par un ẻlargissement des domaines touchant la recherche et une ouverture à desservir une clientèle externe autre que les gestionnaires mêmes de Tourisme Canada.

Sur le plan du contenu, le programme de recherche, initialement centré sur le développement de modèles typiquement économiques tels les prévisions, les mesures d'impacts, le maintien d'inventaires et la collecte de données statistiques, $s^{+} e s t$ élargi pour porter sur des études touchant la demande de tourisme dans des marchés spécifiques. Notons, à ce titre, l'étude sur le marché américain des voyages d'agrément, laquelle, par le biais de 9000 entrevues approfondies, a permis de cerner l'étendue, la composition, les prédilections et les préconceptions sur le Canada de nos voisins américains.

La mise en place d'un programme structuré d'études de marché a constitué l'amorce d'un mouvement sur la nécessité de regrouper divers intervenants afin de canaliser les ressources financières, pour réaliser les projets de recherche. Le partnership s'est done installé. Les recherches de marché, réalisées conjointement avec le USTTA dans 13 pays, illustrent cette approche.

\section{Le marketing}

Au plan du marketing, les changements n'en ont pas moins été importants. En 1985-1986, Tourisme Canada abandonnait totalement ses efforts de marketing sur le marché canadien, concentrant ses ressources sur le développement des marchés américains et d'outre-mer. La priorité était ainsi donnée à l'apport de bénéfices économiques nets pour le Canada via le tourisme international plutôt que la redistribution des revenus touristiques entre les provinces et territoires.

Forts des nouvelles données foumies par l'étude sur le marché américain, une nouvelle stratégie et un plan de marketing ambitieux ont êté mis en oeuvre sur le marché des États-Unis. La campagne $\mathrm{L} e$ Canada: un monde à votre porte est née. Elle avait pour but d'accroître la connaissance du Canada et de créer une image Canada en tant que destination, en mettant en valeur trois facettes importantes du Canada: Le paradis naturel, Les traditions européennes et Le Canada ultramoderne. Cette dernière facette était nouvelle et visait à corriger les perceptions des américains; seloneux, le Canada était ennuyeux et offrait peu de possibilités de divertissement.

Sur les marchés d'outre-mer, les efforts de marketing sesont concentrés exclusivement sur quatre marchés: la Grande-Bretagne, l'Allemagne de l'ouest, le Japon et la France. Les stratégies et les plans de marketing sur ces marchés ont été ajustés au cours des ans pour saisir des possibilités de marchés importants comme la croissance rapide du marché japonais et pour élargir la gamme des produits offerts sur ces marchés. De plus, des alliances se sont créces entre les provinces (ex.: Canada-Québec-Ontario sur le marché japonais) et avec certains transporteurs aériens. De nouvelles approches ont été adoptées en ce qui concerne la participation aux foires et aux bourses de voyage.

La consultation de 1985 avait révélé une lacune importante au plan de lacoordination des efforts de marketing, des gouvernements et de l'industrie. C'est de là qu'est né le Conseil de la commercialisation touristique qui regroupe les responsables de marketing des provinces et des territoires de même que les entreprises qui consacrent 500
$000 \$$ et plus en marketing sur les marchés internationaux. Le Conseil est devenu un forum de discussions et $d^{*}$ échanges d'information sur les stratégies et plans de marketing des différents partenaires; il permet d'accroittre l'harmonisation, la coordination et la coopération.

\section{Le développement du produit}

Durant les années ' 70 , nous avons assisté à la mis en place de grandes infrastructures et d'équipements majeurs qui sont venus appuyer l'offre touristique. Nous n'avons qu'à penser au réseau routier, aux infrastructures aéroportuaires, aux nouveaux musées, aux parcs naturels, aux sites historiques... Les programmes directs d'appui au développement touristique ont suivi cette tendance. La première entente Canada-Québec sur le développement touristique, conclue en 1978 , a permis d'investir dans des equipements publics tels les parcs provinciaux, la restauration de bâtiments historiques, le réseau des bases de plein air, le palais des congrès de Montreal.

La deuxième génération d'ententes fé́dérales-provinciales conclues en 1984 et 1985 s"est démarquée de la précédente en privilégiant:

- l'amélioration de la compétitivité internationale de $l^{\text {'industrie touristique }}$ canadienne:

- un rồle-clé pour le secteur privé dans le développement du produit;

- la promotion de l'investissement.

Au Québec,où la plus importante des douze ententes a été conclue, la priorité a été accordée à un appui au secteur privé dans la modernisation des stations touristiques de ski alpin et au développement de centres de villégiatures quatre-saisons dans les régions des Laurentides, des Cantons de l'Est et du Québec.

Autre facteur intéressant: les programmes d'aide a l'industrie, traditionnellement à $l^{\prime}$ intention des entreprises manufacturières, ont commencé à élargir leurs critères d'admissibilité pour inclure les entreprises touristiques. Le programme de développement industriel régional(PDIR), le programme Action Atlantique et le PDET en sont des exemples.

D'autre part, au plan des grandes politiques qui ont eu un impact sur le tourisme, le mouvement de déréglementation du transport aérien a certes été, au cours des 
années ' 80 , celle qui a le plus marqué le tourisme international.

\section{La situation de l'industrie et l'évolution de ses marchés}

Avant de situer l'intervention du gouvernement fédéral en matière de tourisme dans les années ${ }^{7} 90$, il y a lieu de se demander oùenest l'industrie canadienne du tourisme et quels sont les principaux defis auxquels elle doit faire face.

Avec ses 24 milliards $\$$ de revenus en 1988 , le secteur touristique représente $4 \%$ du P.N.B.. Il emploie 5, $1 \%$ de la main-d'oeuvre active au Canada ( 630000 emplois divers). Il est la deuxième source de devises étrangères (7 milliards \$) après l'industrie del'automobile. La croissance del'industrie a été constante au cours des 5 dernières années (6,7\% par année entre 1984 et 1988) et ce sont les recettes touristiques internationales qui sont responsables de cette croissance. Leur part des recettes totales est passée de $22,7 \%$ à $28,2 \%$ entre 1986 et 1988.

Malgré cette performance, le tourisme est un des secteurs industriels les moins reconnus au Canada. De fait, très peu de dirigeants et encore moins de canadiens réalisent l'importance du tourisme sur notre économie nationale. Ils ont tendance à prendre sa croissance pour acquise.

Bien que les résultats finals ne soient pas compilés, il est d'ores et dejà acquis que le rythme de croissance connu au cours des cinq dernières années s'est ralenti en 1989. Les américains sont venus en moins grand nombre qu'en 1988 (moins 4,4\%) et la croissance des touristes venant d'outre-mer $\mathrm{s}^{7}$ est poursuivie $(7,6 \%)$ mais à un rythme plus lent.

Le ralentissement Economique aux EtatsUnis n'explique pas à lui seul cette performance de l'industrie en 1989. Nous commençons à réaliser l'impact de changements structurels au niveau du marché. Lesegment de marché traditionnel rubber tire se rétrécit. De plus, les américains sont agressivement sollicités par d'autres destinations internationales (Europe, Australie, Mexique, Asie) et les états americains consacrent des budgets importants pour convaincre les résidents des états voisins de découvrir leur coin de pays.

Notre clientèle touristique est en évolution. Les voyageurs internationaux viennent surtout des pays industrialisés du monde occidental et ces pays font face an un vieillissement marqué de leur population, à des familles moins nombreuses, à un plus grand nombre de familles monoparentales et à une urbanisation généralisée.

Dans leur ensemble, les gens du groupe d'âge 55 à 64 ans ont plus de loisirs, sont en meilleure santé et disposent d'un revenu discrétionnaire plus élevé. Ce grouped'âge va grandissant. Les familles à double gagnepain sont monnaie courante et disposent d'un revenu discrétionnaire pour les voyages. Toutefois, il est difficile de faire coincider les calendriers pour de grandes vacances et par conséquent elles préferent faire un plus grand nombre de courts voyages.

Les communications modernes font du monde un endroit plus exigu et moins déroutant. Les voyages lointains ne font plus peur. Les images a l'écran, les affaires à l'échelle globale, les transports de plus en plus faciles d'accès sont autant d'incitations à voyager de par le monde, ce que font un nombre grandissant de personnes. Le raffinement des voyageurs a d'autres répercussions. Avec une meilleure education et un revenu plus éleve, ils recherchent une meilleure qualité de service et une "expérience" plus stimulante.

Voyageurs plus chevronnés gråce à une retraite plus hâtive, à une meilleure santé et à un revenu discrétionnaire plus imposant, ils deviennent plusexigeants et ils réclament la diversité. Il faut donc constamment se renouveler pour les attirer.

Les marchés non traditionnels, notamment le Sud-Est asiatique, croissent en importance. Cette tendance découle de deux facteurs: la puissance économique nouvellement acquise de ces pays et une population canadienne en évolution. Devenus plus riches, les gens de certains pays asiatiques sont maintenant plus mobiles. Les immigrants en provenance de l'Inde et du reste de l'A Asie rendent le Canada plus visible dans leur pays $\mathrm{d}^{4}$ origine et leur présence ici attire leurs parents et amis en visite chez nous.

\section{Des problèmes à résoudre}

Dans le court et le moyen terme, la pénurie de travailleurs est une des questions les plus pressantes quiconfrontent $1^{\dagger}$ industrie. Parce qu'elle a mauvaise presse auprès des gens qui accèdent au marché du travail (salaire inférieur et longues heures de travail) et parce qu'elle fait appel à un réservoir de jeunes gens dont le nombre est en diminution au moment où l'industrie est en plein essor, 1 'industrie touristique rencontre des difficultés plus grandes à recruter des employés que bien d'autres secteurs économiques. Une formation inadéquate, un taux de roulement élevé et une mobilité ascendante et latérale restreinte constituent également une entrave au recrutement. RÉsultat: l'industrie souffre d'une carence de travailleurs bien formés dans de nombreux domaines étroitement liés à la qualité du service, de même que dans le domaine de la gestion touristique.

Commec'est le cas pour toutes les industries modernes, le tourisme s'épanouira ou diminuera selon la qualité des renseignements dont il dispose. Le succès dans l'élaboration, la conception, la promotion et la vente de produits dépend de la qualité des renseignements dont nous disposerons sur les nouvelles tendances, sur les agissements de nos concurrents et sur les marchés en général.

L'accès à un financement suffisant constitue un élément essentiel à la mise en place de nouvelles entreprises touristiques. Dans une certaine mesure, les projets touristiques ont encore mauvaise réputation dans les milieux financiers à cause de déboires passés, d'un pauvre retour sur l'investissementetd'une courte saisond'exploitation. Les avantages que comporte l'industrie, par exemple la croissance soutenue du marché, les risques réels d'investissement et un plus grand professionnalisme en gestion, sont mal compris et ne suffisent toujours pas à attirer les fonds requis.

La mise en forfait d'une expérience touristique est une autre constituante essentielle sur laquelle doit se pencher l'industrie. A l'heure actuelle, l'industrie touristique a une approche fragmentaire et incohérente face à la mise en forfait des voyages. Les divers secteurs de l'industrie - hotels, restaurants, attractions diverses. lignes aériennes, sociétés de chemin de fer et $d^{4}$ autobus - poursuivent leurs propres objectifs indépendamment les uns des autres, sans souci des avantages communs qui découlent d'une planification globale bien orchestrée.

Les changements qui s"opèrent au niveau des marchés offrent la possibilité de tirerun meilleur parti de toutes les saisons. Cependant, il va falloir coordonner de nombreux facteurs commerciaux et opérationnels: la tarification, l'organisation des voyages, le recrutement du personnel 
et, enfin, les politiques gouvemementales qui limitent encore la saison d'ouverture des installations publiques.

L'adaptation de nouvelles technologies au sein des entreprises touristiques permettra de faire des gains de productivité. La globalisation des systèmes de distribution qui offrira la possibilité de rejoindre un plus grand nombre de clients, l'intégration des lignes aériennes, le climat de déréglementation, le besoin d'augmenter les capacités sur certaines routes aériennes et celui de développer de nouvelles liaisons sur des destinations touristiques prometteuses, sont autant de facteurs qui influenceront la croissance du tourisme international au Canada.

La libéralisation des échanges commerciaux créera des possibilités mais aura aussi un impact sur la façon de brasser des affaires. La globalisation des économies et l'augmentation des voyages d'affaires qui en résultera aura un impact sur le tourisme international. L'équilibre entre le développement touristique et la protection du milieu naturel et de la qualité de vie des communautés demeurera un défi constant.

S'il est vrai que l'industrie touristique connaît une croissance rapide partout dans le monde, il est également vrai que la concurrence pour les dollars touristiques existants et futurs s'intensifie. Les industries de service constituent le secteuréconomique qui connaît la plus forte croissance dans le monde occidental. Tous les gouvernements en sont venus à reconnaître l'importance du tourisme au sein du secteur des services.

\section{L'intervention fédérale dans les années '90}

Les gouvernements ont été et continueront d'être partie intégrante de l'industrie touristique. Ils ont joué un rôle important dans la définition des réalités touristiques. De fait, les gouvernements, à tous les niveaux, sont directement engages dans l'industrie canadienne du tourisme soit à titre de propriétaires, soit en accordant les permis ou en régissant le fonctionnement des installations et des services qui composent l'infrastructure touristique. Cet engagement ne se limite pas aux parcs et aux sites historiques mais s'étend à une vaste gamme d'installations de transport, d'accords internationaux, de mesures fiscales et $\mathrm{d}^{+}$autres politiques.

L'État demeurera présent mais son intervention devra se situer à un niveau qui lui permettra d'être le plus efficace et le plus efficient dans une conjoncture globale en pleine ébullition. Cette intervention sera conditionnée également par les forces et les limites des produits touristiques canadiens, de même que par les problèmes ou contraintes qui entravent le développement

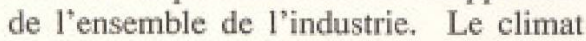
fiscaldu gouvernement fédéral va influencer son action auprès de l'industrie touristique canadienne.

Tous ces facteurs militent en faveur d'un virage où la recherche, l'information, la définition des cibles, la capacité de réconcilier des intérêts disparates pour la réalisation d'un but commun et une vision des moyens à prendre pour réaliser les possibilités identifiées seront les grandes lignes de force de l'action gouvernementale.

Déjà amorcée, la politique visant à s'éloigner de l'intervention directe par des subventions individuelles à des entreprises se poursuivra et amènera Tourisme Canada à se diriger vers:

1- un rỏle d'intercesseur plus fort afin d'exercer une influence sur les politiques et sur les programmes du gouvernement fédéral qui ont des retombées sur le développement touristique. Les questions de ressources humaines, les questions d'investissement, de financement et de fiscalité ainsi que le cadre réglementaire qui les régit, les questions de transport aérien, notamment les accords bilatéraux, les questions relatives au tourisme et à l'environnement devraient constituer un agenda prioritaire sur ce plan.

2- un rôle d'encadrement de l'industrie par la fourniture d'informations et de services à l'industrie tels: la conduite de recherches innovatrices; la mise en disponibilité d 'informations commerciales à joury compris des données sur les tendances des marchés et des produits sur la demande et sur la concurrence ainsi que des analyses de la performance de l'industrie; l'utilisation d'informations et d'analyses pour la formulation de stratégies et de plans, que ce soit au niveau d'une région, d'une destination, d'un produit; la dissémination de cette information par le biais de séminaires, de missions ou de publications: l'établissement de réseaux de communication; la coordination d'un développement continuel de produits touristiques visant les marchés à forte croissance et à rendement fort et exploitant les quatre saisons; l'incitation à une plus grande utilisation de la technologie.

3- uneprésence internationale plus ciblée et plus efficace qui favorise une prise de conscience du Canada comme destination touristique dans certains marchés étrangers et qui assure une coordination des efforts de marketing international des partenaires clés de l'industrie touristique. Les efforts de marketing du gouvemement fédéral devraient porter sur:

- les segments de marché à haut rendement et à forte croissance comme cible:

+ la promotion des saisons autres que l'été et des produits à haut rendement;

- l'augmentation de l'intensité des efforts de marketing auprès des consommateurs haut-de-gamme (ceux qui dépensent le plus), des personnes de l'âge d'or, des voyageurs hors saison et de fin de semaine et des voyageurs de long parcours;

- la promotion des produits sousutilisés qui, selon les résultats de la recherche, présentent des possibilités;

- le mariage de nouvelles initiatives de marketing à la conception de produits innovateurs comme le voyage d'évasion, l'ensemble urbainrural, le voyage d'aventure plein-air et l'expérience de culture autochtone.

\section{Conclusion}

Au-delà des rêves, il y a les réalités économiques, sociales et commerciales du tourisme. Plus que jamais, tous les partenaires de l'industrie touristique canadienne devront travailler ensemble et former une coalition réelle pour faire face aux défis qu'amèneront la globalisation des économies, la liberralisation des échanges commerciaux, le relâchement des tensions internationales, ladétermination des peuples à se développer de façon compatible avec leur environnement.

Reconnaître l'importance de l'industrie touristique pour l'économie du pays n'est plus suffisant. Il faudra considérer cette industrie comme un secteur à part entière dans les plans stratégiques et économiques des gouvernements. 